to develop and maintain networks of nontraditional service providers to compensate for this lack of access; however, many agencies are unaware of each others' roles in maintaining the health of the community. A novel method of building and evaluating mental health service networks was trailed in rural New South Wales. Methods: Workshops providing education and support for agencies involved in providing services to rural communities were held in 12 locations. Participants' knowledge of mental health issues and the role of other support agencies, and their confidence in using this knowledge, were evaluated by pre- and postworkshop surveys. A new method of evaluating existing network strength and efficacy, using structured key informant interviews and network analysis methods, was trailed in several locations. Local service networks were reevaluated following the workshops to determine the extent, direction and determinants of network change. Results: About 96 workshop participants completed pre- and postworkshop surveys. Significant improvements in knowledge, confidence and social distance were found. Local referral networks were successfully mapped and changes in local communities following the workshops were evaluated.

Conclusions: Existing networks of service providers in rural communities have substantial capacity to act in nontraditional ways to improve the mental health of those communities. A relatively simple intervention, combined with ongoing support from local health agencies, can increase this capacity and the strength and efficiency of networks.

\section{A taxometric exploration of alcohol and cannabis problems in a community sample}

\section{T Slade', M Teesson ${ }^{2}$}

'University of New South Wales; and ${ }^{2}$ National Drug and Alcohol Research Centre, Sydney, Australia

Background: There is growing evidence that problems associated with substance use disorders (eg dependence and abuse) are most appropriately conceptualized using one or more continuous dimensions rather than as categorical 'yes/no' entities. Taxometric analysis is a statistical technique specifically designed to test this assertion.

Method: DSM-IV symptoms associated with the use of alcohol and cannabis dependence and abuse were subjected to a taxometric analysis using data from a large epidemiological survey of mental disorders in the general population.

Results: Alcohol use problems were best explained by a latent, continuous dimension ranging from mild to severe, while problems associated with using cannabis were best explained by a latent discrete category.

Conclusions: These findings have implications for the way that substance use problems are measured and classified and show specificity with regard to the type of substance under investigation.

\section{Diagnosing mild cognitive impairment: the problem with subjective complaints}

\author{
M Slavin, H Brodaty, N Kochan, P Sachdev
}

The University of New South Wales, Sydney, Australia

Subjective cognitive complaints (SCCs) are common in older adults with up to $88 \%$ of people aged over 85 years complaining of memory problems. The concept of SCCs is ill defined even though they comprise the first of Petersen's diagnostic criteria for mild cognitive impairment (MCI). There is little agreement on how to operationalize SCCs - is it sufficient to simply ask 'have you noticed problems with your memory or thinking?' Nonmemory cognitive complaints have been relatively ignored. The criterion is sensitive but not specific because SCCs are so common. Evidence suggests that SCCs correlate poorly with objective cognitive impairments. It is therefore important to examine the most useful way to define SCCs and whether they contribute significantly to the diagnosis of MCI. We are currently conducting a community study of memory and aging, during which each participant completes three different measures of SCC - two focused on memory and one that covers other cognitive domains. We present data from 300 participants aged $70-90,35 \%$ of whom have cognitive impairment on neuropsychological testing. Cognitive impairment was defined as a result 1.5 SDs or more below normal for age. Cognitive complaints were common in our group - with each measure of SCC being affirmed by up to $66 \%$ of participants. We confirmed a lack of correlation between SCCs and objective cognitive impairment. No one measure of SCC was shown to be more accurate at predicting objective impairment than another. In conclusion, we question whether it is appropriate to preserve $\mathrm{SCC}$ as a diagnostic criterion for MCI.

\section{Collective trauma: the case for a multilevel social-ecological perspective}

\section{Somasundaram \\ University of Adelaide, Adelaide, Australia}

Background: Complex emergencies that follow war and natural disasters have an impact on not only the 